\title{
MIURA TYPE TRANSFORMATIONS AND HOMOGENEOUS SPACES
}

\author{
SERGEY A. IGONIN
}

\begin{abstract}
We relate Miura type transformations (MTs) over an evolution system to its zero-curvature representations with values in Lie algebras $\mathfrak{g}$. We prove that certain homogeneous spaces of $\mathfrak{g}$ produce MTs and show how to distinguish these spaces. For a scalar translation-invariant evolution equation this allows to classify all MTs in terms of homogeneous spaces of the WahlquistEstabrook algebra of the equation. For other evolution systems this allows to construct some MTs. As an example, we study MTs over the $\mathrm{KdV}$ equation, a 5th order equation of Harry-Dym type, and the coupled KdV-mKdV system of Kersten and Krasilshchik.
\end{abstract}

\section{INTRODUCTION}

In this paper we study $(1+1)$-dimensional evolution systems

$$
\begin{gathered}
\frac{\partial v^{i}}{\partial t}=R^{i}\left(x, t, v^{1}, \ldots, v^{k}, v_{1}^{1}, \ldots, v_{1}^{k}, \ldots, v_{r}^{1}, \ldots, v_{r}^{k}\right), \\
\frac{\partial u^{i}}{\partial t}=P^{i}\left(x, t, u^{1}, \ldots, u^{k}, u_{1}^{1}, \ldots, u_{1}^{k}, \ldots, u_{p}^{1}, \ldots, u_{p}^{k}\right), \\
v_{j}^{i}=\frac{\partial^{j} v^{i}}{\partial x^{j}}, \quad u_{j}^{i}=\frac{\partial^{j} u^{i}}{\partial x^{j}}, \quad i=1, \ldots, k,
\end{gathered}
$$

and transformations

$$
u^{i}=S^{i}\left(x, t, v^{1}, \ldots, v^{k}, v_{1}^{1}, \ldots, v_{1}^{k}, \ldots, v_{n}^{1}, \ldots, v_{n}^{k}\right)
$$

such that if $v^{1}, \ldots, v^{k}$ satisfy (11) then (3) satisfy (2). By analogy with the classical Miura transformation connecting the $\mathrm{KdV}$ and $\mathrm{mKdV}$ equations, such transformations are called Miura type transformations (MTs in short).

It is well known that MTs play an important role in the theory of integrable evolution systems. Chains of MTs generate Bäcklund transformations [13, 14.

1991 Mathematics Subject Classification. 37K35, 53C30.

Key words and phrases. Evolution systems, Miura type transformations, homogeneous spaces, Wahlquist-Estabrook algebras, the KdV equation. 
If $P^{i}, R^{i}, S^{i}$ do not depend on $x$ and $t$, the MT is called translationinvariant.

In the scalar case $k=1$, the problem to find all pairs (2), (3) for a given system (11) was solved efficiently in [16].

We study the opposite problem: for a given system (2), how to find all pairs (11), (3) ? It seems that this problem was systematically studied only for translation-invariant MTs of the linear equation $u_{t}=u_{3}$, and the KdV equation [2, 3, 9]. In particular, a set of MTs over the $\mathrm{KdV}$ equation was constructed using homogeneous spaces of certain Lie groups (in [2]) and loop groups (in [3]). These MTs do not exhaust all translation-invariant MTs over the KdV equation, but, knowing all integrable equations of the form $u_{t}=u_{3}+f\left(u, u_{1}, u_{2}\right)$, one can show that all other MTs can be obtained from these by introduction of a potential.

We relate MTs (3) with zero-curvature representations of (21) (ZCRs in short) dependent on $x, t, u_{j}^{i}, j \leq p-1$. It turns out that such a ZCR with values in a Lie algebra $\mathfrak{g}$ and a certain representation of $\mathfrak{g}$ by vector fields on a manifold $W$ determine a MT. Among other requirements, the image of $\mathfrak{g}$ under the representation must span the tangent spaces of $W$, that is, the manifold $W$ is a homogeneous space of $\mathfrak{g}$.

This construction is not surprising if one recalls the theory of coverings of PDEs [12, 6]. It is becoming clear [6] that each covering is determined by a $\mathfrak{g}$-valued ZCR and a vector field representation of some Lie algebra $\mathfrak{g}$. Since MTs are a particular type of coverings, it remains to determine which ZCRs and representations lead to MTs. However, in order to be self-contained, we do not introduce the coverings terminology and work in local coordinates.

If $k=1$ and (2) is translation-invariant, we prove that every MT arises in this way from the "universal" ZCR with values in the Wahlquist-Estabrook algebra of (2). This allows to reduce classification of MTs to the classification of certain homogeneous spaces of the Wahlquist-Estabrook algebra. As an example, we obtain that any (not necessarily translation-invariant) MT over the KdV equation is of order not greater than 3. Also, we recover the Lie groups of [2] as the Lie groups associated with some quotients of the Wahlquist-Estabrook algebra and explain why the method of [2] does not give all translation-invariant MTs over the KdV equation.

Another considered example is the equation $u_{t}=u^{\frac{5}{2}} u_{5}$ [4, 11]. Using its Wahlquist-Estabrook algebra computed in 4, we show that any MT over this equation is of order not greater than 5 and construct a 
MT of order 3. The corresponding modified equation (39) may be a new integrable equation.

For nonscalar systems we obtain only a sufficient condition for a ZCR to define a MT. As an example, we construct a MT over the coupled KdV-mKdV system of Kersten and Krasilshchik [8]. This MT arises from the ZCR obtained in [7. Again, the corresponding modified system may be new.

There are also more general transformations of evolution equations, where one changes not only dependent variables, but also the $x$ variable (see, e.g., 5, 16, 15]). It remains an interesting open question whether our Lie algebraic methods can be generalized for studying of these transformations.

The paper is organized as follows. In Section 2 we study Lie algebras actions of special type that will later turn out to be responsible for MTs. In Sections 3, 4we describe MTs of scalar evolution equations and their relations with Wahlquist-Estabrook algebras. Finally, in Section 5 we study MTs of nonscalar evolution systems.

\section{Actions of Lie Algebras on manifolds}

Recall that an action of a Lie algebra $\mathfrak{g}$ on a manifold $W$ is a homomorphism $\rho: \mathfrak{g} \rightarrow D(W)$ to the Lie algebra $D(W)$ of vector fields on $W$. The action is said to be transitive if for each point $a \in W$ the mapping

$$
\mathrm{ev}_{\rho, a}: \mathfrak{g} \rightarrow T_{a} W, \quad g \mapsto \rho(g)_{a},
$$

is surjective. Two actions $\rho_{i}: \mathfrak{g} \rightarrow D\left(W_{i}\right), i=1,2$, are said to be isomorphic if there is a diffeomorphism $\varphi: W_{1} \rightarrow W_{2}$ such that $\rho_{2}=$ $\varphi_{*} \rho_{1}$.

Below our considerations are always local. The results are valid in both categories of smooth and complex-analytic manifolds. Depending on the category considered, all functions are supposed to be smooth or complex-analytic.

In what follows we often consider a (possibly infinite) chain of subalgebras

$$
\mathfrak{g}^{1} \subset \mathfrak{g}^{2} \subset \mathfrak{g}^{3} \subset \cdots \subset \mathfrak{g}
$$

and an action $\rho: \mathfrak{g} \rightarrow D(W)$. It easy to see that there is a non-empty open subset $W_{c} \subset W$ such that

$$
\begin{gathered}
\forall a, a^{\prime} \in W_{c}, \quad \forall i \quad \operatorname{dim} \operatorname{ev}_{\rho, a}\left(\mathfrak{g}^{i}\right)=\operatorname{dim}_{\rho, a^{\prime}}\left(\mathfrak{g}^{i}\right), \\
\operatorname{dim} \operatorname{ev}_{\rho, a}(\mathfrak{g})=\operatorname{dim}_{\rho, a^{\prime}}(\mathfrak{g})
\end{gathered}
$$


Moreover, if $W$ is connected and $\rho$ is analytic, one can choose $W_{c}$ to be dense in $W$. Since we study locally nondegenerate points only, below in the paper we always assume $W=W_{c}$.

Denote $m_{i}=\operatorname{dimev}_{\rho, a}\left(\mathfrak{g}^{i}\right)$ for $a \in W_{c}$. Due to inclusions (41) we have

$$
m_{1} \leq m_{2} \leq m_{3} \leq \ldots
$$

Lemma 1. In the above described situation, suppose that there is $V \in$ $D(W)$ such that for each $i$ the Lie algebra generated by the subspace $\rho\left(\mathfrak{g}^{i}\right)+\left[\rho\left(\mathfrak{g}^{i}\right), V\right]$ coincides with $\rho\left(\mathfrak{g}^{i+1}\right)$. Set $n=\operatorname{dim} W$. Suppose that

$$
m_{1} \geq s, \quad m_{n-s}<n, \quad \exists k \quad m_{k}=n .
$$

Then

$$
m_{i}=s+i-1, \quad i=1, \ldots, n-s+1 .
$$

Moreover, for each point $z \in W$ on a neighborhood of $z$ there is a function $w$ such that

$$
d_{z} w \neq 0, \quad \rho\left(\mathfrak{g}^{n-s}\right)(w)=0,
$$

and this function is unique up to a change $w \mapsto g(w)$. Set

$$
\tilde{w}^{i}=V^{i-1}(w), \quad i=1, \ldots, n-s+1 .
$$

One can find functions $\tilde{w}^{n-s+2}, \ldots, \tilde{w}^{n}$ such that $\tilde{w}^{1}, \ldots, \tilde{w}^{n}$ form a system of coordinates on a neighborhood of $z$.

Proof. For a set $S$ of vector fields denote by $\langle S\rangle$ the submodule (over the algebra of functions) of vector fields generated by $S$. By condition (5) and the Frobenius theorem, there are coordinates $w^{1}, \ldots, w^{n}$ on a neighborhood of $z$ such that

$$
\forall i \quad\left\langle\rho\left(\mathfrak{g}^{i}\right)\right\rangle=\left\langle\frac{\partial}{\partial w^{1}}, \ldots, \frac{\partial}{\partial w^{m_{i}}}\right\rangle .
$$

Denote module (9) by $M_{i}$. Suppose that $m_{k}=m_{k+1}$ for some $k$. Then $\left[M_{k}, V\right] \subset M_{k}$ and, therefore, $m_{p}=m_{k}$ for all $p \geq k$. Combining this property with (17) and (6), we obtain (8).

Now we can take $w=w^{n}$. Using the equalities

$$
M_{i}+\left\langle\left[M_{i}, V\right]\right\rangle=M_{i+1}, \quad i=1, \ldots, n-s,
$$

by induction on $i$ one proves

$$
M_{n-s-i+1}\left(\tilde{w}^{i}\right)=0, \quad \frac{\partial \tilde{w}^{i}}{\partial w^{n-i+1}} \neq 0, \quad i=1, \ldots, n-s+1,
$$

where

$$
M_{0}=\left\langle\frac{\partial}{\partial w^{1}}, \ldots, \frac{\partial}{\partial w^{s-1}}\right\rangle
$$


Therefore, if we set $\tilde{w}^{n-s+1+i}=w^{i}$ for $i=1, \ldots, s-1$, the functions $\tilde{w}^{1}, \ldots, \tilde{w}^{n}$ will be local coordinates on a neighborhood of $z$.

\section{MTs FROM ZCRs of SCALAR EVOLUTION EQUATIONS}

Consider two scalar evolution equations

$$
\begin{array}{ll}
u_{t}=P\left(x, t, u, u_{1}, \ldots, u_{p}\right), & u_{k}=\frac{\partial^{k} u}{\partial x^{k}}, \\
v_{t}=R\left(x, t, v, v_{1}, \ldots, v_{r}\right), & v_{k}=\frac{\partial^{k} v}{\partial x^{k}}
\end{array}
$$

connected by a MT

$$
u=S\left(x, t, v, v_{1}, \ldots, v_{n}\right) .
$$

The maximal integer $n$ such that (12) depends nontrivially on $v_{n}$ is called the order of the MT. A MT obtained from this one by a substitution $v \mapsto g(v)$ is said to be equivalent to the initial MT.

Introduce new variables

$$
w^{i}=\frac{\partial^{i-1} v}{\partial x^{i-1}}, \quad i=1, \ldots, n,
$$

and rewrite system (11), (12) as follows

$$
\begin{aligned}
\frac{\partial w^{i}}{\partial x} & =w^{i+1}, \quad i=1, \ldots, n-1, \\
\frac{\partial w^{n}}{\partial x} & =a\left(w^{1}, \ldots, w^{n}, x, t, u\right), \\
\frac{\partial w^{i}}{\partial t} & =b^{i}\left(w^{1}, \ldots, w^{n}, x, t, u, \ldots, u_{p-1}\right), \quad i=1, \ldots, n,
\end{aligned}
$$

where $p$ is the order of (10). And vice versa, it is easily seen that any consistent system of this form with the non-degeneracy condition

$$
\exists w_{0}^{1}, \ldots, w_{0}^{n}, x_{0}, t_{0}, u_{0} \quad \frac{\partial a}{\partial u}\left(w_{0}^{1}, \ldots, w_{0}^{n}, x_{0}, t_{0}, u_{0}\right) \neq 0
$$

determines a MT of order $n$ for (10) as follows:

- substitute (13) to (14),

- taking into account (15), from equation (14) express locally $u=S\left(x, t, v, v_{1}, \ldots, v_{n}\right)$,

- let $D=\sum_{i \geq 0} v_{i+1} \partial / \partial v_{i}$, then equation (11) is given by

$$
v_{t}=b^{1}\left(v, v_{1}, \ldots, v_{n-1}, x, t, S, D(S), \ldots, D^{p-1}(S)\right) \text {. }
$$


Consider the total derivative operators

$$
\begin{aligned}
D_{x} & =\frac{\partial}{\partial x}+\sum_{j \geq 0} u_{j+1} \frac{\partial}{\partial u_{j}}, \\
D_{t} & =\frac{\partial}{\partial t}+\sum_{j \geq 0} D_{x}^{j}\left(P\left(x, t, u, u_{1}, \ldots, u_{p}\right)\right) \frac{\partial}{\partial u_{j}}
\end{aligned}
$$

and more general overdetermined systems

$$
\begin{aligned}
& \frac{\partial w^{i}}{\partial x}=a^{i}\left(w^{1}, \ldots, w^{n}, x, t, u\right), \quad i=1, \ldots, n, \\
& \frac{\partial w^{i}}{\partial t}=b^{i}\left(w^{1}, \ldots, w^{n}, x, t, u, \ldots, u_{p-1}\right), \quad i=1, \ldots, n,
\end{aligned}
$$

consistent modulo (10). Clearly, an invertible change of variables

$$
w^{i} \mapsto f^{i}\left(w^{1}, \ldots, w^{n}\right)
$$

leads to a new system of the form (16). Two systems related by such a change of variables are said to be equivalent.

System (16) is completely determined by the vector fields

$$
\begin{gathered}
A=\sum_{i=1}^{n} a^{i}\left(w^{1}, \ldots, w^{n}, x, t, u\right) \frac{\partial}{\partial w^{i}}, \\
B=\sum_{i=1}^{n} b^{i}\left(w^{1}, \ldots, w^{n}, x, t, u, \ldots, u_{p-1}\right) \frac{\partial}{\partial w^{i}} .
\end{gathered}
$$

Consistency of (16) modulo (10) is equivalent to the equation

$$
\left[D_{x}+A, D_{t}+B\right]=0 .
$$

Recall that two functions

$$
M(x, t, u), \quad N\left(x, t, u, \ldots, u_{p-1}\right)
$$

with values in a Lie algebra $\mathfrak{g}$ constitute a zero-curvature representation (ZCR in short) for (10) if

$$
\left[D_{x}+M, D_{t}+N\right]=D_{x} N-D_{t} M+[M, N]=0 .
$$

Then each action $\rho: \mathfrak{g} \rightarrow D(W)$ and a choice of local coordinates $w^{1}, \ldots, w^{n}$ in $W$ determine a consistent system of the form (16) with $A=\rho(M)$ and $B=\rho(N)$, since equation (18) follows from (20). Clearly, different choices of coordinates in $W$ or isomorphic actions determine equivalent systems (16).

Definition 1. Suppose that a ZCR (19) is given. For each $k \in \mathbb{N}$ we define a subalgebra $\mathfrak{g}^{k}$ of $\mathfrak{g}$ by induction on $k$ as follows:

$$
\text { - } \mathfrak{g}^{0}=0,
$$


- $\mathfrak{g}^{1}$ is the subalgebra generated by all elements

$$
M(x, t, u)-M\left(x^{\prime}, t^{\prime}, u^{\prime}\right) \in \mathfrak{g},
$$

where $x, t, u, x^{\prime}, t^{\prime}, u^{\prime}$ run through all admissible (real or complex) values of the corresponding variables.

- $\mathfrak{g}^{k+1}$ is generated by the subspaces $\mathfrak{g}^{k}$ and $\left[\mathfrak{g}^{k}, M(x, t, u)\right]$.

Remark 1. Note that due to the definition of $\mathfrak{g}^{1}$ the space

$$
\mathfrak{g}^{k}+\left[\mathfrak{g}^{k}, M(x, t, u)\right]
$$

does not depend on the values of $x, t, u$.

Set also $\tilde{\mathfrak{g}}=\cup_{k \geq 0} \mathfrak{g}^{k}$.

Theorem 1. Suppose that system (16) arises from an action $\rho: \mathfrak{g} \rightarrow$ $D(W)$. Then the following two statements are equivalent.

(1) There are $z=\left(w_{0}^{1}, \ldots, w_{0}^{n}\right) \in W$ and an invertible transformation (17) on a neighborhood of z such that system (16) takes the form (14) with (15).

(2) There are $z \in W$ and a neighborhood $W_{0}$ of $z$ such that

$$
\begin{gathered}
\operatorname{dim} \operatorname{ev}_{\rho, z}(\tilde{\mathfrak{g}})=n, \\
\forall a \in W_{0} \quad \operatorname{dim} \operatorname{ev}_{\rho, a}\left(\mathfrak{g}^{n-1}\right)<n, \\
\exists x_{0}, t_{0}, u_{0} \quad \frac{\partial}{\partial u}(\rho(M))\left(z, x_{0}, t_{0}, u_{0}\right) \neq 0 .
\end{gathered}
$$

In this case on a neighborhood of $z$ there is a function $w$ such that

$$
d_{z} w \neq 0, \quad \rho\left(\mathfrak{g}^{n-1}\right)(w)=0,
$$

and it is unique up to a change $w \mapsto g(w)$. The functions

$$
\tilde{w}^{i}=\rho(M(x, t, u))^{i-1}(w), \quad i=1, \ldots, n,
$$

do not depend on $x, t, u$ and are local coordinates in which system (16) takes the desired form (14), (15).

Proof. If system (16) is of the form (14), (15) then by the definition of $\mathfrak{g}^{k}$ we obtain that on a neighborhood of $z$ the image of $\mathfrak{g}^{k}$ in each tangent space of $W$ is spanned by $\partial / \partial w^{n-k+1}, \ldots, \partial / \partial w^{n}$. This obviously implies (21) and (22), and (23) follows from (15).

Conversely, let (21), (22), and (23) hold. Then existence of $w$ and the fact that $\tilde{w}^{1}, \ldots, \tilde{w}^{n}$ are local coordinates follow from Lemma 1 for $s=1$ and $V=\rho(M(x, t, u))$. In particular, the functions $\tilde{w}^{1}, \ldots, \tilde{w}^{n-1}$ are invariant under $\rho\left(\mathfrak{g}^{1}\right)$. Combining this fact with the formula $\tilde{w}^{k+1}=$ $\rho(M(x, t, u))\left(\tilde{w}^{k}\right)$, by induction on $k$ one gets that each function $\tilde{w}^{k}$ does not depend on $x, t, u$. 
It is easily seen that system (16) is of the form (14) in the coordinates $\tilde{w}^{1}, \ldots, \tilde{w}^{n}$. Finally condition (15) follows from (23).

Remark 2. In the above theorem, to construct a MT it is sufficient to know the restriction of $\rho$ to some neighborhood of $z$, and condition (21) implies that the action $\left.\rho\right|_{\tilde{\mathfrak{g}}}$ is transitive on a neighborhood of $z$.

Corollary 1. If $\mathfrak{g}^{m}=\mathfrak{g}^{m+1}$ for some $m \geq 0$ (equivalently, $\tilde{\mathfrak{g}}=\mathfrak{g}^{m}$ ) then actions of $\mathfrak{g}$ cannot produce MTs of order greater than $\mathrm{m}$.

Proof. By Theorem 1 and the above remark, a MT of order $n$ is determined by a transitive action $\rho$ of $\tilde{\mathfrak{g}}$ such that $\rho\left(\mathfrak{g}^{n-1}\right)$ is not transitive. Since in our case $\tilde{\mathfrak{g}}=\mathfrak{g}^{k}$ for any $k \geq m$, there are no such actions for $n>m$.

Remark 3. If $M$ does not depend on $x, t$ then (23) follows from (21) and (22). Indeed, from the above proof in this case $\operatorname{dim} \operatorname{ev}_{\rho, z}\left(\mathfrak{g}^{1}\right)=1$, which implies that

$$
\exists u_{0} \quad \frac{\partial \rho(M)}{\partial u}\left(z, u_{0}\right) \neq 0 .
$$

\section{WAhlquist-Estabrook ALgebras And MTs}

4.1. General results. For a scalar translation-invariant equation

$$
u_{t}=P\left(u, u_{1}, \ldots, u_{p}\right)
$$

recall the definition of the Wahlquist-Estabrook algebra [18] from the point of view of [12]. Consider the equation

$$
\left[D_{x}+A(u), D_{t}+B\left(u, u_{1}, \ldots, u_{p-1}\right)\right]=0,
$$

where $A, B$ are functions with values in a (not specified in advance) Lie algebra $\mathfrak{g}$. The algebra $\mathfrak{g}$ can also be the algebra of vector fields on a manifold $W$, then $A$ and $B$ are vector fields on $W$ dependent on $u, \ldots, u_{p-1}$. Suppose that for any $\mathfrak{g}$ equation (27) implies

$$
\begin{gathered}
A=\sum_{i=1}^{k_{1}} f_{i}(u) F_{i}, \\
B=\sum_{i=1}^{k_{2}} g_{i}\left(u, u_{1}, \ldots, u_{p-1}\right) G_{i},
\end{gathered}
$$

where $f_{i}, g_{i}$ are some fixed scalar functions, which do not depend on $\mathfrak{g}$, and $F_{i}, G_{i}$ are elements of $\mathfrak{g}$. Moreover, suppose that functions (28) satisfy (27) if and only if some Lie algebra relations hold between the elements $F_{1}, \ldots, F_{k_{1}}, G_{1}, \ldots, G_{k_{2}}$. In this case the quotient of the free Lie algebra generated by the letters $F_{i}, G_{j}$ over these relations is called 
the Wahlquist-Estabrook algebra of (26) and is denoted $\mathfrak{w}$. Functions (28), (29) constitute a ZCR with values in we such that any consistent translation-invariant system (16) arises from this ZCR and some action of $\mathfrak{w e}$.

The Wahlquist-Estabrook algebra exists for practically all known equations (see, e.g., [1, 12 and references therein).

By Theorem 11 and Remarks 2, 3, we obtain the following result.

Theorem 2. Translation-invariant MTs of order $n$ of (26) are in oneto-one correspondence with actions $\rho$ of $\mathfrak{w e}$ such that $\rho(\tilde{\mathfrak{w}} \mathfrak{e})$ is transitive and $\rho\left(\mathfrak{m} \mathfrak{e}^{n-1}\right)$ is not transitive. Locally isomorphic actions determine equivalent MTs.

It turns out that non-translation-invariant MTs can also be described in terms of actions of $\mathfrak{w e}$. By the construction of [10, with any system (14) we can associate the following translation-invariant system

$$
\begin{aligned}
\frac{\partial \hat{w}^{1}}{\partial x} & =\frac{\partial \hat{w}^{2}}{\partial t}=1, \quad \frac{\partial \hat{w}^{2}}{\partial x}=\frac{\partial \hat{w}^{1}}{\partial t}=0, \\
\frac{\partial w^{i}}{\partial x} & =w^{i+1}, \quad i=1, \ldots, n-1, \\
\frac{\partial w^{n}}{\partial x} & =a\left(w^{1}, \ldots, w^{n}, \hat{w}^{1}, \hat{w}^{2}, u\right), \\
\frac{\partial w^{i}}{\partial t} & =b^{i}\left(w^{1}, \ldots, w^{n}, \hat{w}^{1}, \hat{w}^{2}, u, \ldots, u_{p-1}\right), \quad i=1, \ldots, n .
\end{aligned}
$$

This system is consistent provided that the initial system (14) is consistent and equation (10) (equation (26)) is translation-invariant. It is determined by an action $\rho$ of $\mathfrak{w} \mathfrak{e}$ on the manifold with coordinates $\hat{w}^{1}, \hat{w}^{2}, w^{1}, \ldots, w^{n}$. If system (14) arises from a MT (12), (111) then we have (15), which similarly to the proof of Theorem 1 implies

$$
\operatorname{dim} \operatorname{ev}_{\rho, a}(\tilde{\mathfrak{w} \mathfrak{e}})=n, \quad \operatorname{dim} \operatorname{ev}_{\rho, a}\left(\mathfrak{w} \mathfrak{e}^{n-1}\right)=n-1 .
$$

This observation implies the following (compare with Corollary 1).

Theorem 3. If for some $n$ we have $\mathfrak{m}^{n}=\tilde{\mathfrak{w}} \mathfrak{e}$ then any (not necessarily translation-invariant) MT over (26) is of order not greater than $n$.

\subsection{MTs of the $\mathbf{K d V}$ equation. Consider the $\mathrm{KdV}$ equation}

$$
u_{t}=u_{3}+u_{1} u
$$

in the complex-analytic category. According to [17, we have

$$
\mathfrak{w} \mathfrak{e}=H \oplus \mathfrak{s l}_{2}(\mathbb{C}) \otimes_{\mathbb{C}} \mathbb{C}[\lambda]
$$


where $H$ is the 5-dimensional nilpotent Heisenberg algebra with the basis $r_{i}, i=-2,-1,0,1,2$, and the commutator table

$$
\left[r_{-1}, r_{1}\right]=\left[r_{2}, r_{-2}\right]=r_{0}, \quad\left[r_{i}, r_{j}\right]=0 \forall i+j \neq 0
$$

and the Lie bracket in $\mathfrak{s l}_{2} \otimes \mathbb{C}[\lambda]$ is defined as follows $\left[g_{1} \otimes f_{1}(\lambda), g_{2} \otimes f_{2}(\lambda)\right]=\left[g_{1}, g_{2}\right] \otimes f_{1}(\lambda) f_{2}(\lambda), \quad g_{i} \in \mathfrak{s l}_{2}, f_{i}(\lambda) \in \mathbb{C}[\lambda]$.

Below an element $g \otimes f(\lambda)$ of $\mathfrak{s l}_{2} \otimes \mathbb{C}[\lambda]$ will be written simply as $g f(\lambda)$.

The universal ZCR reads

$$
\begin{gathered}
A(u)=X_{1}+\frac{1}{3} u X_{2}+\frac{1}{6} u^{2} X_{3} \\
X_{1}=r_{1}-\frac{1}{2} y+\frac{1}{2} z \lambda, \quad X_{2}=r_{-1}+z, \quad X_{3}=r_{-2},
\end{gathered}
$$

where $h, y, z$ is a basis of $\mathfrak{s l}_{2}$ with the relations $[h, y]=2 y,[h, z]=$ $-2 z,[y, z]=h$. Here the form of $B\left(u, u_{1}, u_{2}\right)$ in (29) is not important for us.

From (30) and (31), using Definition 11, one obtains

$$
\begin{gathered}
\mathfrak{w} \mathfrak{e}^{1}=\left\langle X_{2}, X_{3}\right\rangle, \quad \mathfrak{w}^{2}=\left\langle r_{-2}, r_{-1}, z, 2 r_{0}+h\right\rangle, \\
\mathfrak{w} \mathfrak{e}^{3}=\mathfrak{w} \mathfrak{e}^{k}=\tilde{\mathfrak{w}} \mathfrak{e}=\left\langle\mathfrak{s l} l_{2} \otimes \mathbb{C}[\lambda], r_{-2}, r_{-1}, r_{0}\right\rangle \quad \forall k \geq 3 .
\end{gathered}
$$

By Theorem 3, any MT of the KdV equation is of order not greater than 3. For translation-invariant MTs this was proved in 9 .

Let us explain how our method of constructing MTs includes the one of [2]. Set $\mathfrak{g}=\mathfrak{s l}_{2}(\mathbb{C}) \otimes_{\mathbb{C}} \mathbb{C}[\lambda]$. We have the natural projection $\mathfrak{w} \rightarrow \mathfrak{g}$ that maps $H$ to zero. Combining it with the above ZCR, we obtain a ZCR with values in $\mathfrak{g}$ whose $x$-part reads

$$
M(u)=-\frac{1}{2} y+\frac{1}{2} z \lambda+\frac{1}{3} u z .
$$

For this ZCR we have

$$
\mathfrak{g}^{1}=\langle z\rangle, \quad \mathfrak{g}^{2}=\langle z, h\rangle, \quad \mathfrak{g}^{3}=\tilde{\mathfrak{g}}=\mathfrak{g} .
$$

By Theorem 2] each transitive action $\rho$ of $\mathfrak{g}=\tilde{\mathfrak{g}}$ on a manifold of dimension $n \leq 3$ determines a MT for the KdV equation, because the algebra $\rho\left(\mathfrak{g}^{n-1}\right)$ is of dimension $\leq n-1$ and cannot be transitive.

According to [6], for a transitive action $\rho: \mathfrak{g} \rightarrow D(W)$ the image $\rho(\mathfrak{g})$ is finite-dimensional and is of the form

$$
\mathfrak{s l}_{2} \otimes \mathbb{C}[\lambda] /(f(\lambda)), \quad f(\lambda) \in \mathbb{C}[\lambda],
$$

where $(f(\lambda))$ is the ideal of $\mathbb{C}[\lambda]$ generated by $f(\lambda)$. Let

$$
f(\lambda)=a \prod_{s=1}^{k}\left(\lambda-e_{s}\right)^{k_{s}}, \quad a, e_{s} \in \mathbb{C}, \quad a \neq 0, \quad e_{i} \neq e_{j} \forall i \neq j .
$$


Then Lie algebra (32) is isomorphic to

$$
\bigoplus_{s=1}^{k} \mathfrak{s l}_{2} \otimes \mathbb{C}[\lambda] /\left(\lambda^{k_{s}}\right) .
$$

The Lie groups $\prod_{s} \mathrm{SL}_{2}\left(\mathbb{C}[\lambda] /\left(\lambda^{k_{s}}\right)\right)$ that appear in 2] have (33) as their Lie algebras. Thus construction of MTs arising from this ZCR is reduced to local description of homogeneous spaces of dim $\leq 3$ of these Lie groups. This description and the corresponding MTs are presented in 2 .

A translation-invariant MT over the $\mathrm{KdV}$ equation belongs to the list of MTs in [2] if and only if for the corresponding action $\rho$ of $\mathfrak{w e}$ we have $\rho(H)=0$.

Example 1. Consider the following action of $\mathfrak{w} \mathfrak{e}$ on $\mathbb{C}$

$$
\mathfrak{g} \rightarrow 0, \quad r_{i} \mapsto 0, i=-2,0,1,2, \quad r_{-1} \mapsto \frac{\partial}{\partial w},
$$

where $w$ is a coordinate in $\mathbb{C}$. The corresponding MT is

$$
u=3 v_{1}, \quad v_{t}=v_{3}+\frac{3}{2} v_{1}^{2}
$$

and does not belong to the list of MTs in [2].

4.3. MTs of a Harry-Dym type equation. Consider the equation [4, 11]

$$
u_{t}=u^{5 / 2} u_{5}
$$

in the complex-analytic category. There are Bäcklund transformations connecting (34) with the Sawada-Kotera and Kaup-Kupershmidt equations [4.

According to 4, the Wahlquist-Estabrook algebra of (34) is the direct sum $\mathbb{C}^{2} \oplus \mathfrak{g}$, where $\mathbb{C}^{2}$ is a commutative algebra with a basis $C_{1}, C_{2}$ and $\mathfrak{g}$ is the "positive part" of the twisted affine algebra $A_{2}^{(2)}$. In other words, the algebra $\mathfrak{g}$ is isomorphic to a subalgebra of $\mathfrak{s l}_{3}(\mathbb{C}) \otimes_{\mathbb{C}}$ $\mathbb{C}[\lambda]$ generated by the two elements

$$
X_{1}=\left\|\begin{array}{ccc}
0 & 0 & 0 \\
\lambda & 0 & 0 \\
0 & 0 & 0
\end{array}\right\|, \quad X_{2}=\left\|\begin{array}{ccc}
0 & 0 & 1 \\
0 & 0 & 0 \\
0 & 1 & 0
\end{array}\right\|
$$

satisfying the relations

$$
\begin{aligned}
& \left(\operatorname{ad} X_{1}\right)^{2} X_{2}=0 \\
& \left(\operatorname{ad} X_{2}\right)^{5} X_{1}=0
\end{aligned}
$$


The corresponding $\mathrm{ZCR}$ reads

$$
A(u)=C_{1} u^{-\frac{1}{2}}+X_{1} u^{-\frac{3}{2}}+X_{2},
$$

the form of $B\left(u, u_{1}, u_{2}, u_{3}, u_{4}\right)$ can be found in [4 and is not important for our purposes.

The subalgebra $\mathfrak{w e}^{k}, k \geq 1$, is generated by the elements

$$
C_{1}, \quad\left(\operatorname{ad} X_{2}\right)^{i} X_{1}, \quad i=0, \ldots, k-1 .
$$

Combining this with relation (37), we obtain $\mathfrak{w}^{5}=\mathfrak{w} \mathfrak{e}^{6}=\tilde{\mathfrak{w}} \mathfrak{e}$. By Theorem 3, we obtain that any MT over (34) is of order not greater than 5 .

Let us construct a MT over (34). Consider the homomorphism $\mathfrak{w} \mathfrak{e} \rightarrow$ $\mathfrak{s l}_{3}$ that maps $C_{i}$ to zero and substitutes $\lambda=1$ in (35). Combining the standard action of $\mathfrak{s l}_{3}$ on $\mathbb{C}^{3}$ with this homomorphism, we obtain the following transitive action

$$
\begin{gathered}
\rho: \mathfrak{w} \mathfrak{e} \rightarrow D\left(\mathbb{C}^{3}\right), \quad \rho\left(C_{i}\right)=0, \\
\rho\left(X_{1}\right)=w^{2} \frac{\partial}{\partial w^{1}}, \quad \rho\left(X_{2}\right)=w^{3} \frac{\partial}{\partial w^{2}}+w^{1} \frac{\partial}{\partial w^{3}} .
\end{gathered}
$$

According to Theorem 11, to get a MT from this action we need to find a nonconstant function $w$ on $\mathbb{C}^{3}$ such that $\rho\left(\mathfrak{r e}^{2}\right)(w)=0$. Since the algebra $\rho\left(\mathfrak{m e}^{2}\right)$ is commutative and spanned by the vector fields

$$
\rho\left(X_{1}\right)=w^{2} \frac{\partial}{\partial w^{1}}, \quad \rho\left(\left[X_{2}, X_{1}\right]\right)=w^{3} \frac{\partial}{\partial w^{1}}-w^{2} \frac{\partial}{\partial w^{3}},
$$

we can take $w=w^{2}$. By formula (25) for $M(x, t, u)=A(u)$, we have $\tilde{w}^{1}=w^{2}, \tilde{w}^{2}=w^{3}, \tilde{w}^{3}=w^{1}$. Rewriting the vector field $\rho(A(u))$ in these coordinates, we obtain that the $x$-part of the corresponding system (14) is

$$
\frac{\partial \tilde{w}^{1}}{\partial x}=\tilde{w}^{2}, \quad \frac{\partial \tilde{w}^{2}}{\partial x}=\tilde{w}^{3}, \quad \frac{\partial \tilde{w}^{3}}{\partial x}=u^{-\frac{3}{2}} \tilde{w}^{1} .
$$

Applying the substitution (13) for $w^{i}=\tilde{w}^{i}$, from (38) we obtain the MT

$$
u=\left(\frac{v}{v_{3}}\right)^{\frac{2}{3}}
$$

The corresponding equation (11) can be obtained either by straightforward computation or using the vector field $\rho\left(B\left(u, u_{1}, u_{2}, u_{3}, u_{4}\right)\right)$ as described in Section 3. The answer is

$$
v_{t}=-9 v D^{2}\left(\left(\frac{v}{v_{3}}\right)^{\frac{2}{3}}\right)+\frac{9}{2} v_{1} D\left(\left(\frac{v}{v_{3}}\right)^{\frac{2}{3}}\right)-\frac{3}{2} v_{2}\left(\frac{v}{v_{3}}\right)^{\frac{2}{3}},
$$

where $D=\sum_{i} v_{i+1} \partial / \partial v_{i}$. 


\section{MTS OF NONSCALAR EVOLUTION SYSTEMS}

5.1. MTs from ZCRs. A MT (3) of nonscalar systems cannot always be written in some simple analog of the form (14), and, therefore, not all MT of nonscalar systems can be described by our method. In this section we study the MTs that can be written in a form analogous to (14).

Consider an evolution system (2) and a system

$$
\begin{gathered}
\frac{\partial w^{j}}{\partial x}=w^{i+1}, \quad j=1, \ldots, n-s, \\
\frac{\partial w^{n-s+i}}{\partial x}=A^{i}\left(w^{1}, \ldots, w^{n}, x, t, u^{1}, \ldots, u^{k}\right), \quad i=1, \ldots, s, \\
\frac{\partial w^{l}}{\partial t}=b^{i}\left(w^{1}, \ldots, w^{n}, x, t, u^{1}, \ldots, u^{k}, u_{1}^{1}, \ldots, u_{1}^{k}, \ldots\right), \\
l=1, \ldots, n .
\end{gathered}
$$

consistent modulo (2). By analogy with (15), suppose that the following condition holds

$$
\begin{gathered}
\exists w_{0}^{1}, \ldots, w_{0}^{n}, x_{0}, t_{0}, u_{0}^{1}, \ldots, u_{0}^{k} \\
\operatorname{rank}\left\|\frac{\partial A^{i}}{\partial u^{j}}\right\|\left(w_{0}^{1}, \ldots, w_{0}^{n}, x_{0}, t_{0}, u_{0}^{1}, \ldots, u_{0}^{k}\right)=s .
\end{gathered}
$$

In particular, from (43) we have $s \leq k$.

Then one gets a MT over (2) as follows. Taking into account (43), by the implicit function theorem, there are $1 \leq j_{1}<\cdots<j_{s} \leq k$ such that $u^{j_{1}}, \ldots, u^{j_{s}}$ can locally be expressed from (41) in terms of

$$
x, t, \quad u^{n_{1}}, \ldots, u^{n_{k-s}}, \quad w^{1}, \ldots, w^{n}, \quad \frac{\partial w^{n-s+i}}{\partial x}, \quad i=1, \ldots, s,
$$

where $n_{1}<n_{2}<\cdots<n_{k-s}$ are such that

$$
\left\{j_{1}, \ldots, j_{s}, n_{1}, \ldots, n_{k-s}\right\}=\{1, \ldots, k\} .
$$

Substitute everywhere

$$
\begin{gathered}
w^{j}=v_{j-1}^{1}, \quad \frac{\partial w^{n-s+1}}{\partial x}=v_{n-s}^{1}, \quad j=1, \ldots, n-s, \\
w^{n-s+j}=v^{j}, \quad \frac{\partial w^{n-s+j}}{\partial x}=v_{1}^{j}, \quad j=2, \ldots, s, \\
u^{n_{j}}=v^{s+j}, \quad j=1, \ldots, k-s .
\end{gathered}
$$

Thus we expressed $u^{i}$ in terms of $x, t, v_{l}^{j}$, that is, we got (3). Finally, system (11) is obtained from (42). 
The total derivative operators are now

$$
\begin{aligned}
D_{x} & =\frac{\partial}{\partial x}+\sum_{\substack{i=1, \ldots, k, j \geq 0}} u_{j+1}^{i} \frac{\partial}{\partial u_{j}^{i}}, \\
D_{t} & =\frac{\partial}{\partial t}+\sum_{\substack{i=1, \ldots, k, j \geq 0}} D_{x}^{j}\left(P^{i}\left(x, t, u, u_{1}, \ldots, u_{p}\right)\right) \frac{\partial}{\partial u_{j}^{i}} .
\end{aligned}
$$

Similarly to (16) we consider systems

(44)

$$
\begin{aligned}
& \frac{\partial w^{i}}{\partial x}=a^{i}\left(w^{1}, \ldots, w^{n}, x, t, u^{1}, \ldots, u^{k}\right), \quad i=1, \ldots, n, \\
& \frac{\partial w^{i}}{\partial t}=b^{i}\left(w^{1}, \ldots, w^{n}, x, t, u^{1}, \ldots, u^{k}, u_{1}^{1}, \ldots, u_{1}^{k}, \ldots\right), \quad i=1, \ldots, n .
\end{aligned}
$$

consistent modulo (2) with the same equivalence relation (17).

Two functions

$$
M\left(x, t, u^{1}, \ldots, u^{k}\right), \quad N\left(x, t, u^{1}, \ldots, u^{k}, u_{1}^{1}, \ldots, u_{1}^{k}, \ldots\right)
$$

with values in a Lie algebra $\mathfrak{g}$ constitute a ZCR for (2) if equation (20) holds. Each action $\rho: \mathfrak{g} \rightarrow D(W)$ and a choice of local coordinates $w^{1}, \ldots, w^{n}$ in $W$ determine a consistent system of the form (44) as follows

$$
\begin{gathered}
\rho(M)=\sum_{i=1}^{n} a^{i}\left(w^{1}, \ldots, w^{n}, x, t, u^{1}, \ldots, u^{k}\right) \frac{\partial}{\partial w^{i}} \\
\rho(N)=\sum_{i=1}^{n} b^{i}\left(w^{1}, \ldots, w^{n}, x, t, u^{1}, \ldots, u^{k}, u_{1}^{1}, \ldots, u_{1}^{k}, \ldots\right) \frac{\partial}{\partial w^{i}} .
\end{gathered}
$$

Similarly to Definition [1] we define subalgebras $\mathfrak{g}^{k}$ of $\mathfrak{g}$ by induction on $k$ as follows:

- $\mathfrak{g}^{0}=0$,

- $\mathfrak{g}^{1}$ is the subalgebra generated by all elements

$$
M\left(x, t, u^{1}, \ldots, u^{k}\right)-M\left(x^{\prime}, t^{\prime},\left(u^{1}\right)^{\prime}, \ldots,\left(u^{k}\right)^{\prime}\right) \in \mathfrak{g},
$$

- $\mathfrak{g}^{k+1}$ is generated by the subspaces $\mathfrak{g}^{k}$ and

$$
\left[\mathfrak{g}^{k}, M\left(x, t, u^{1}, \ldots, u^{k}\right)\right] .
$$

Set also $\tilde{\mathfrak{g}}=\cup_{k \geq 0} \mathfrak{g}^{k}$.

Theorem 4. Suppose that system (44) arises from an action $\rho: \mathfrak{g} \rightarrow$ $D(W)$ and there are $x_{0}, t_{0}, u_{0}^{1}, \ldots, u_{0}^{k}, z \in W$ and a neighborhood $W_{0}$ 
of z such that

$$
\begin{gathered}
\operatorname{dim} \operatorname{ev}_{\rho, z}(\tilde{\mathfrak{g}})=n, \\
\forall a \in W_{0} \quad \operatorname{dim} \operatorname{ev}_{\rho, a}\left(\mathfrak{g}^{n-s}\right)<n,
\end{gathered}
$$

where $s$ is the dimension of the subspace

$$
\left\langle\frac{\partial \rho(M)}{\partial u^{i}}\left(z, x_{0}, t_{0}, u_{0}^{1}, \ldots, u_{0}^{k}\right) \mid i=1, \ldots, k\right\rangle \subset T_{z} W .
$$

Then on a neighborhood of $z$ there is a function $w$ such that

$$
d_{z} w \neq 0, \quad \rho\left(\mathfrak{g}^{n-s}\right)(w)=0,
$$

and it is unique up to a change $w \mapsto g(w)$. The functions

$$
\tilde{w}^{i}=\rho\left(M\left(x, t, u^{1}, \ldots, u^{k}\right)\right)^{i-1}(w), \quad i=1, \ldots, n-s+1,
$$

do not depend on $x, t, u^{i}$. One can find functions $\tilde{w}^{n-s+2}, \ldots, \tilde{w}^{n}$ such that $\tilde{w}^{1}, \ldots, \tilde{w}^{n}$ form a system of coordinates on a neighborhood of $z$. The initial system (16) takes the form (40), (41), (42), (43) in these coordinates.

Proof. Similarly to Theorem 1, this follows from Lemma 1 for $V=$ $\rho\left(M\left(x, t, u^{1}, \ldots, u^{k}\right)\right)$. Condition $m_{1}=\operatorname{dimev}_{\rho, z}\left(\mathfrak{g}^{1}\right) \geq s$ holds because the space $\operatorname{ev}_{\rho, z}\left(\mathfrak{g}^{1}\right)$ includes subspace (45).

5.2. MTs of the Kersten-Krasilshchik system. The following system was introduced in [8] and proved to be integrable

$$
\begin{aligned}
& u_{t}^{1}=-u_{3}^{1}+6 u^{1} u_{1}^{1}-3 u^{2} u_{3}^{2}-3 u_{1}^{2} u_{2}^{2}+3 u_{1}^{1}\left(u^{2}\right)^{2}+6 u^{1} u^{2} u_{1}^{2}, \\
& u_{t}^{2}=-u_{3}^{2}+3\left(u^{2}\right)^{2} u_{1}^{2}+3 u^{1} u_{1}^{2}+3 u_{1}^{1} u^{2} .
\end{aligned}
$$

Denote by $E_{i j}, i, j=1,2,3$, the $(3 \times 3)$-matrix with $(i, j)$-entry equal to 1 and other entries equal to 0 . Let $\mathfrak{g}$ be the 5 -dimensional Lie subalgebra of $\mathfrak{s l}_{3}(\mathbb{C})$ spanned by the elements

$$
e=E_{12}, \quad n_{1}=E_{13}, \quad f=E_{21}, \quad n_{2}=E_{23}, \quad h=E_{11}-E_{22} .
$$

An $\mathfrak{s l}_{3}$-valued ZCR dependent on a parameter $\lambda$ was constructed for (46) in [7]. For $\lambda=0$ one obtains the following ZCR

$$
M=\left(\left(u^{2}\right)^{2}-u^{1}\right) e-f-u^{2} n_{1}
$$

and

$$
\begin{aligned}
& N=\left(u_{2}^{1}+u^{2} u_{2}^{2}+\left(u_{1}^{2}\right)^{2}-2\left(u^{1}\right)^{2}+\left(u^{2}\right)^{4}+\left(u^{2}\right)^{2} u^{1}\right) e \\
& -\left(u_{1}^{1}+u^{2} u_{1}^{2}\right) h-\left(\left(u^{2}\right)^{2}+2 u^{1}\right) f+\left(u_{2}^{2}-\left(u^{2}\right)^{3}-2 u^{1} u^{2}\right) n_{1}+u_{1}^{2} n_{2} .
\end{aligned}
$$


Let us construct MTs for (46) from this ZCR using Theorem 4. We have

$$
\begin{gathered}
\mathfrak{g}^{1}=\left\langle e, n_{1}\right\rangle, \\
\mathfrak{g}^{2}=\left\langle h, e, n_{1}, n_{2}\right\rangle, \quad \mathfrak{g}^{3}=\tilde{\mathfrak{g}}=\mathfrak{g} .
\end{gathered}
$$

Every subalgebra $\mathfrak{h} \subset \mathfrak{g}$ of codimension 3 such that

$$
\mathfrak{g}^{1} \cap \mathfrak{h}=0
$$

determines a MT as follows. Consider Lie groups $H \subset G$ corresponding to the Lie algebras $\mathfrak{h} \subset \mathfrak{g}$ and set $W=G / H, z=H \in G / H$. We have the natural transitive action of $\rho: \mathfrak{g} \rightarrow D(W)$. From (47) and (48) we obtain that the dimension of space (45) is equal to 2 . Since $\operatorname{dim} \mathfrak{g}^{1}=2$ and $\operatorname{dim} W=3$, the algebra $\rho\left(\mathfrak{g}^{1}\right)$ is not transitive on $W$. Therefore, all conditions of Theorem 4 are satisfied, and $\rho$ determines a MT with $n=3, s=2$.

For example, set $\mathfrak{h}=\left\langle n_{2}, f-n_{1}\right\rangle$. The corresponding action in local coordinates reads

$$
\begin{gathered}
e \mapsto \frac{\partial}{\partial w^{2}}, \quad n_{1} \mapsto \frac{\partial}{\partial w^{3}}, \quad n_{2} \mapsto w^{2} \frac{\partial}{\partial w^{3}} \\
f \mapsto w^{2} \frac{\partial}{\partial w^{1}}-\left(w^{2}\right)^{2} \frac{\partial}{\partial w^{2}}+\left(\mathrm{e}^{-3 w^{1}}-w^{2} w^{3}\right) \frac{\partial}{\partial w^{3}} \\
h \mapsto \frac{\partial}{\partial w^{1}}-2 w^{2} \frac{\partial}{\partial w^{2}}-w^{3} \frac{\partial}{\partial w^{3}}
\end{gathered}
$$

Indeed, the isotropy subalgebra of the point $z=(0,0,0)$ is equal to $\mathfrak{h}$. The MT reads

$$
\begin{gathered}
u^{1}=v_{2}^{1}+\left(v_{1}^{1}\right)^{2}+\left(v_{1}^{2}+v_{1}^{1} v^{2}-\mathrm{e}^{-3 v^{1}}\right)^{2}, \\
u^{2}=v_{1}^{2}+v_{1}^{1} v^{2}-\mathrm{e}^{-3 v^{1}} .
\end{gathered}
$$

The corresponding system (11) is cumbersome and can be obtained from $\rho(N)$ as described in Subsection 5.1.

Acknowledgements. The author thanks V. V. Sokolov for useful discussions.

\section{REFERENCES}

[1] R. Dodd and A. Fordy. The prolongation structures of quasipolynomial flows. Proc. Roy. Soc. London Ser. A 385 (1983), 389-429.

[2] V. G. Drinfeld and V. V. Sokolov. Equations that are related to the Kortewegde Vries equation. Soviet Math. Dokl. 32 (1985), 361-365.

[3] F. Guil and M. Mañas. Homogeneous manifolds and modified KdV equations. J. Math. Phys. 32 (1991), 1744-1749. 
[4] C. Hoenselaers and W. K. Schief. Prolongation structures for Harry Dym type equations and Bäcklund transformations of CC ideals. J. Phys. A: Math. Gen. 25 (1992), 601-622.

[5] N. H. Ibragimov. Transformation groups applied to mathematical physics. D. Reidel Publishing Co., Dordrecht, 1985.

[6] S. Igonin. Coverings and fundamental algebras for partial differential equations. To appear in J. Geom. Phys.; arxiv:nlin.SI/0301042

[7] A. K. Kalkanli, S. Yu. Sakovich and I. Yurduşen. Integrability of KerstenKrasilshchik coupled KdV-mKdV equations: singularity analysis and Lax pair. J. Math. Phys. 44 (2003), 1703-1708; arxiv:nlin.SI/0206046

[8] P. Kersten and J. Krasilshchik. Complete integrability of the coupled KdVmKdV system. Adv. Stud. Pure Math., Vol. 37, 151-171. Math. Soc. Japan, Tokyo, 2002; arxiv:nlin.SI/0010041

[9] S. V. Khabirov. Equivalence of third-order evolution equations with constant separant. (Russian) Dinamika Sploshn. Sredy 64 (1984), 99-120, 166-167.

[10] E. G. Kirnasov. On Wahlquist-Estabrook type coverings over the heat equation. Math. Notes 42 (1987), 732-739.

[11] B. G. Konopelchenko and V. G. Dubrovsky. Some new integrable nonlinear evolution equations in $2+1$ dimensions. Phys. Lett. A 102 (1984), 15-17.

[12] I. S. Krasilshchik and A. M. Vinogradov. Nonlocal trends in the geometry of differential equations. Acta Appl. Math. 15 (1989), 161-209.

[13] C. Rogers and W. K. Schief. Bäcklund and Darboux transformations. Cambridge Univ. Press, Cambridge, 2002.

[14] C. Rogers and W. F. Shadwick. Bäcklund transformations and their applications. Academic Press, New York, 1982.

[15] S. Yu. Sakovich. On Miura transformations of evolution equations. J. Phys. A 26 (1993), 369-373.

[16] V. V. Sokolov. Pseudosymmetries and differential permutations. Funct. Anal. Appl. 22 (1988), 121-129.

[17] H. N. van Eck. A non-Archimedean approach to prolongation theory. Lett. Math. Phys. 12 (1986), 231-239.

[18] H. D. Wahlquist and F. B. Estabrook. Prolongation structures of nonlinear evolution equations. J. Math. Phys. 16 (1975), 1-7.

Department of Mathematics, Utrecht University, P.O. Box 80010, 3508 TA Utrecht, the Netherlands

E-mail address: igonin@mccme.ru 